\title{
Verruca vulgaris laryngis
}

\section{Definition}

Die Verruca vulgaris ist eine Warzengeschwulst der oberen Hautschichten, die auch auf der Schleimhaut und dem Stimmlippenepithel auftreten kann. Sie wird per definitionem durch das humane Papillomvirus (HPV) hervorgerufen. Bei der Verruca vulgaris laryngis im Larynx wird das Papillomvirus nicht immer nachgewiesen.

\section{Fall}

Ein 65-jähriger Rentner, von Beruf Heizungsinstallateur, leidet seit seinem 41. Lebensjahr an wiederkehrender Heiserkeit durch Entzündungen im Kehlkopf. Er ist Nichtraucher und nimmt gegen hohen Blutdruck Dafiro ${ }^{\circledR}$ (ein Kombinationspräparat aus Valsartan und Amlodipin) sowie Verapamil ein und außerdem Pantozol ${ }^{\circledR}$.

Innerhalb der letzten 24 Jahre fanden mehr als 20 Operationen am Kehlkopf mit Probenentnahmen statt. Histologisch wurden pathologische Verhornungen der Schleimhaut festgestellt. Die warzenartigen Verhornungen führten zeitweise zu einer erheblichen Einengung des Atemweges mit Atemnot. Die histologische Diagnose lautete: Verruca vulgaris laryngis. Ein weiterer histologischer Befund lautete: adulte Papillomatose. Die Untersuchung auf HPV war negativ. Malignität bestand nicht. Die Stimme war durch die Erkrankung und die dadurch notwendigen Operationen tonlos

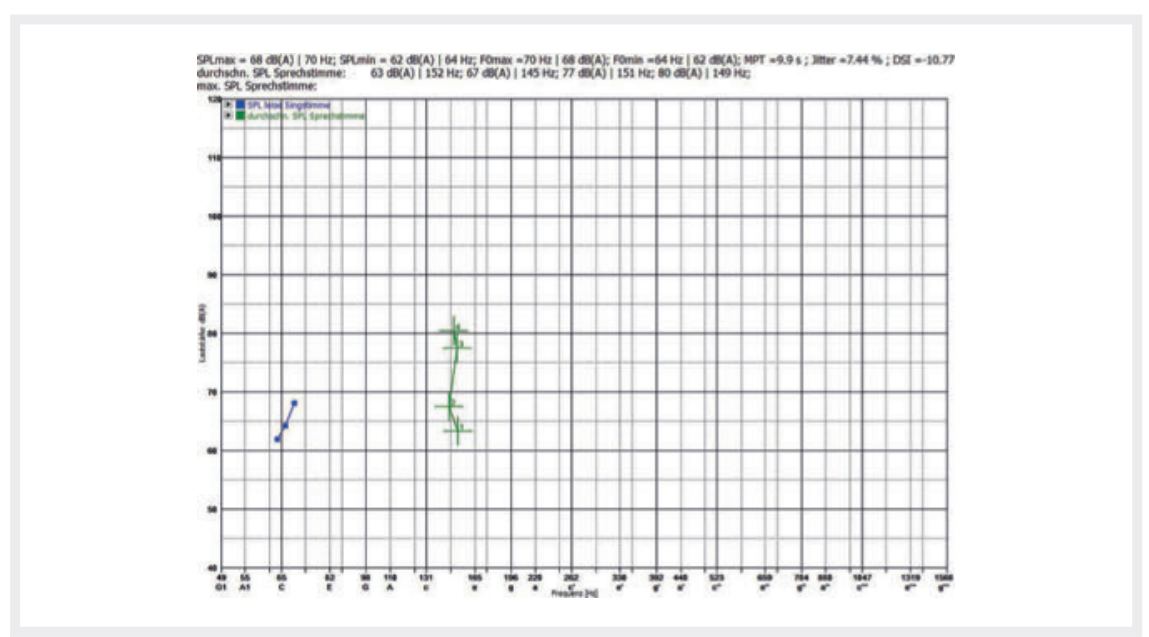

- Abb. 1 Stimmumfangsprofil. Die grünen Kreuze zeigen, welchen Schallpegel der Patient mit seiner Sprechstimme erreicht bei entspanntem Sprechen (unterstes Kreuz) und bei den Versuchen, eine Unterhaltungs-, Vortrags- oder Rufstimme zu erzeugen. Die Stimme ist tonlos, aber das Geräusch lässt sich etwa der Frequenz von ca. $140 \mathrm{~Hz}$ (d) zuordnen. Die drei schwarzen Punkte links bedeuten, dass ein gehaltener Ton um $65 \mathrm{~Hz}$ (C) nur mit einem Pegel von 62-68 dB erzeugt werden kann. (aphon). Trotz sorgfältiger Entfernung aller pathologischen Anteile kam es zu Rezidiven, so dass teilweise in kurzen Intervallen von 2 bis 3 Monaten erneut operiert werden musste.

\section{Befund}

Die gesamte Glottis ist von zottigen weißen Neubildungen der Schleimhaut und des Stimmlippenepithels zirkulär umschlossen ( $\vee$ Video). Die respiratorische Beweglich-

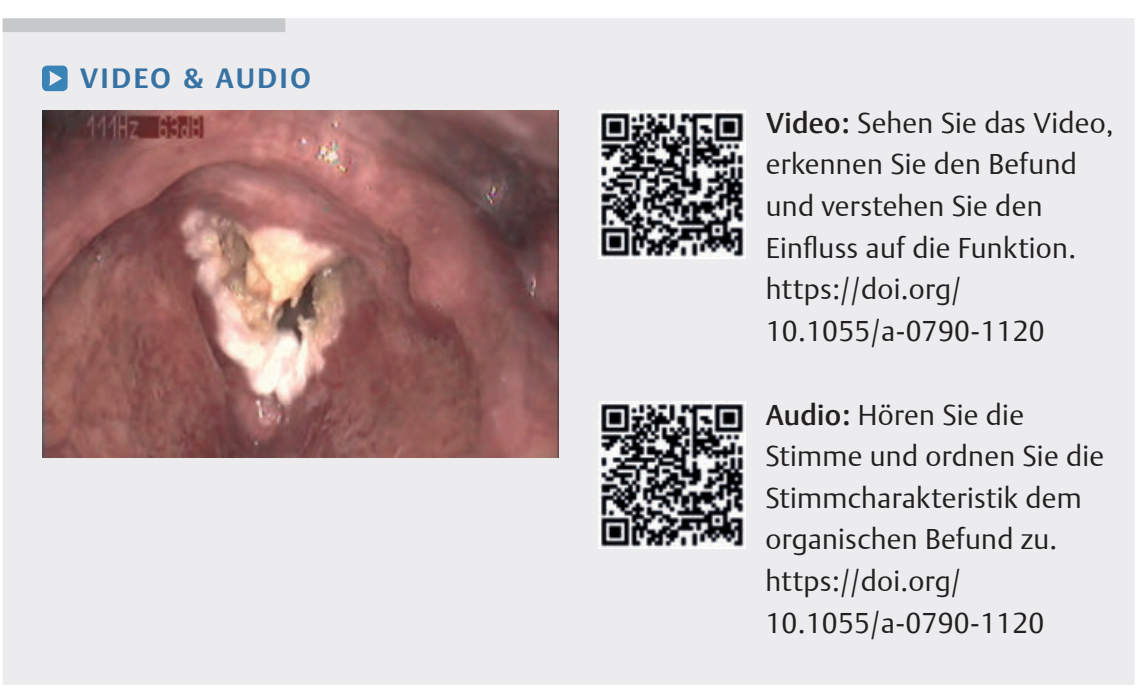

keit ist erhalten. Schwingungsfähiges Gewebe für die Phonation ist nicht mehr vorhanden. Die Stimme ist aphon (\ Audio, Abb. 1).

\section{Ursachen}

Die Verruca vulgaris laryngis ist eine sehr seltene Erkrankung. Bis 2017 wurden dreizehn Fälle in der Literatur beschrieben. Die Ursache ist entweder eine HPV-Infektion (Typen 2, 4, 6 und 11) oder nicht bekannt.

\section{Symptome}

Die exophytischen Warzen haben eine höckrige, weiße Oberfläche, teilweise mit ausgezogenen Zotten. Sie kommen meist auf den Stimmlippen vor, können aber auch auf die Schleimhaut des Larynxinneren übergehen. Die Weite der Glottis wird zuweilen erheblich eingeengt, so dass es zu Atemnot kommen kann. Die Patienten haben eine heisere bis aphone Stimme. Schluckbeschwerden treten in der Regel nicht auf. 


\section{Differenzialdiagnose}

Als Differenzialdiagnose kommen das adulte Papillom, papilläre Keratose und das verruköse Karzinom infrage. Die fransenförmigen (filiformen) Ausziehungen sind nicht pathognomonisch und nicht ausschließlich bei der Verruca vulgaris laryngis zu finden.

\section{Therapie}

Die Behandlung erfolgt in der Regel chirurgisch mit Mikrolaryngoskopie und Laserabtragung. Histologische Untersuchungen müssen sich immer anschließen, damit im Falle einer malignen Entartung die onkologische Therapie frühzeitig begonnen werden kann.

\section{Prognose}

Die Verruca laryngis muss als Präkanzerose angesehen werden. Im hier vorgestellten Fall lag im April des 66. Lebensjahres des Patienten histologisch keine Malignität vor. Im September desselben Jahres wurde ein hochdifferenziertes verruköses Karzinom festgestellt. Der Patient musste schließlich laryngektomiert werden.

FAZIT

Die benigne Verruca laryngis stellt ein Hindernis für die Phonation und die Atmung dar. Die Therapie ist symptomatisch und ermöglicht die histologische Diagnose zum Ausschluss der Malignität.

Interessenkonflikt

Der Autor gibt an, dass kein Interessenkonflikt besteht.

\section{Autorinnen/Autoren}

Tadeus Nawka

Korrespondenzadresse

Prof. Dr. med. Tadeus Nawka

Berlin

E-Mail: Tadeus.Nawka@charite.de
Bibliografie

DOI https://doi.org/10.1055/a-0954-7503

Laryngo-Rhino-Otol 2019; 98: 672-673

(c) Georg Thieme Verlag KG Stuttgart · New York ISSN 0935-8943 